\title{
TIPOS DE CRIANZA DE FELINOS DOMÉSTICOS COMO FACTOR DE RIESGO PARA LA PRESENTACIÓN DE INFECCIÓN POR Toxoplasma gondii
}

\author{
Rearing Conditions in Domestic Feline as a Risk Factor for Toxoplasma \\ GONDII INFECTION
}

Lisset Castillo V. ${ }^{1}$, Norma Noé M. ${ }^{1,3}$, Néstor Falcón P. ${ }^{1,4}$, Amanda Chávez V. ${ }^{2}$

\section{RESUMEN}

El objetivo del estudio fue cuantificar la infección de Toxoplasma gondii en gatos asociado al tipo de crianza: Controlada (sin acceso a la calle) y No controlada (con acceso libre a la calle). Se colectaron 106 muestras de sangre de gatos procedentes de varios distritos de Lima y el nivel de anticuerpos contra T. gondii se analizó con la prueba de Hemaglutinación indirecta. El factor de riesgo para adquirir infecciones por T. gondii reportó un Odds Ratio de 1.2 (0.47 - 3.50). No se encontró asociación significativa entre la frecuencia de infección y el tipo de crianza, pero las variables sexo y dieta mostraron una asociación significativa $(\mathrm{p}<0.05)$. El estudio demuestra que las infecciones por $T$. gondii en gatos no pueden ser atribuidas exclusivamente al tipo de crianza, sino que existen otras fuentes de infección que deben tenerse en cuenta para evitar el riesgo de infección de estos animales y potencialmente del hombre.

Palabras clave: T. gondii, toxoplasmosis, riesgo, tipo de crianza

\section{Abstract}

The objective of the study was to quantify Toxoplasma gondii infections associated to rearing conditions in domestic cats: Controlled (at home) and Uncontrolled (access outside the house). It was collected 106 blood samples from various districts of Lima, and samples were analyzed by the Indirect Haemagglutination test (IHA). The risk factor to acquire T. gondii infection showed an Odds Ratio of 1.2 (0.47 - 3.5). No significant association was found between the frequency of infection and the type of rearing, but variables sex and diet were significantly associated $(\mathrm{p}<0.05)$. The study showed that $T$. gondii infections in domestic cats cannot be entirely attributed to the type of rearing conditions and that other sources of infection have to be taken into account to avoid the risk of infection of these animals and humans.

Key words: Toxoplasma gondii, risk factors, rearing

${ }^{1}$ Laboratorio de Medicina Veterinaria Preventiva, ${ }^{2}$ Laboratorio de Microbiología y Parasitología Veterinaria, Facultad de Medicina Veterinaria, Universidad Nacional Mayor de San Marcos, Lima

${ }^{3}$ E-mail: nvnoemoccetti@gmail.com

${ }^{4}$ Dirección actual: Facultad de Veterinaria y Zootecnia, Universidad Peruana Cayetano Heredia, Lima 


\section{INTRODUCCIÓN}

La toxoplasmosis producida por el Toxoplasma gondii es una zoonosis de distribución mundial que afecta a un alto porcentaje de animales de sangre caliente, incluyendo al hombre (Tenter et al., 2000). La infección es, por lo general, benigna, pero existe un alto riesgo de enfermedad grave $y$ fatal en aquellos individuos con deterioro inmunológico (Montoya y Remington, 2004).

Los felinos juegan un rol importante en la transmisión de $T$. gondii debido a que son los hospederos definitivos y eliminan los ooquistes que contaminan el medio ambiente (Lindsay et al., 1997; Cordero del Campillo et al., 1999). El ratón doméstico y otros roedores pequeños son portadores naturales de T. gondii (Araujo et al., 2003) y las mayores fuentes de infección al gato (Araujo et al., 1998; Dubey y Lappin, 2000), especialmente para aquellos gatos que viven en las calles (Soulsby, 1987; Gauss et al., 2003; Miro et al., 2004). Por ello, el presente estudio tuvo como objetivo cuantificar la asociación entre la variable tipo de crianza de gatos (controlada y no controlada) y la presentación de serorreactores a T. gondii. Ello permitiría inferir, indirectamente, el riesgo de exposición de las personas que se desenvuelven en el entorno de la crianza de estos animales.

\section{Materiales y Métodos}

\section{Tamaño de Muestra y Animales}

Se diseñó un estudio transversal descriptivo considerando dos grupos: gatos de crianza no controlada, con acceso libre a la calle - "gatos techeros" - (Grupo expuesto), y gatos de crianza controlada, criados permanentemente en casa y sin acceso a la calle - "gatos falderos" - (Grupo no expuesto). En general, los gatos de crianza controlada pertenecían a hogares de nivel socioeconómico alto y los de crianza no controlada a un nivel socioeconómico bajo a medio.
La selección de animales no necesariamente siguió un patrón aleatorizado, haciéndose énfasis en el criterio de inclusión (tipo de crianza). La recolección de muestras de sangre de los gatos se realizó entre julio de 2006 a febrero de 2007 en varios distritos de Lima. Se registró información complementaria de cada animal como edad, sexo, dieta y hábitos. El máximo número de gatos muestreado por hogar fue de dos, y en caso hubiera más animales se muestrearon a los dos más dóciles. Los dueños de los animales fueron informados del propósito del estudio y se les entregó los resultados del análisis de las muestras de sus mascotas y las recomendaciones pertinentes al término del trabajo.

El tamaño muestral fue calculado mediante la fórmula de diferencia de proporciones (prueba unilateral) con un nivel de confianza del $95 \%$ y un poder de la prueba del $80 \%$. Se utilizó el valor referencial de un estudio realizado en Valdivia, Chile, donde la prevalencia fue de 33.3\% (Ovalle et al., 2000). Asimismo, considerando que los gatos criados en forma controlada tenían menos probabilidad de infectarse con $T$. gondii, se utilizó el tercio de la prevalencia reportada por Ovalle et al. (2000) en la población general. Es así que se estimó 42 animales como mínimo para cada grupo.

\section{Muestras Biológicas}

Las muestras de sangre se colectaron mediante punción de la vena cefálica. Las muestras se centrifugaron y los sueros se conservaron en congelación a $-20{ }^{\circ} \mathrm{C}$ hasta la determinación de anticuerpos contra T. gondii mediante la técnica de hemaglutinación indirecta (HAI), usando el kit comercial Toxotest HAI ${ }^{\circledR}$ con y sin 2-mercaptoetanol (Wiener Lab). La técnica se basa en la propiedad que tienen los anticuerpos anti-T. gondii de producir aglutinación en presencia de glóbulos rojos sensibilizados con antígenos citoplasmáticos y de membrana del parásito. Se consideraron positivos los títulos $\geq 1 / 16$ (punto de corte) (Wiener Lab, 2000). 
Cuadro 1. Frecuencia de infecciones con Toxoplasma gondii en gatos, según el tipo de crianza, edad, tipo de alimentación, sexo y hábito de caza. Lima, 2007

\begin{tabular}{|c|c|c|c|}
\hline & \multirow{2}{*}{$\begin{array}{c}\text { Animales } \\
\text { muestreados } \\
\text { (n) }\end{array}$} & \multicolumn{2}{|c|}{ Animales positivos } \\
\hline & & $\mathrm{N}^{\mathrm{o}}$ & $\%$ \\
\hline \multicolumn{4}{|l|}{ Tipo de crianza $(\mathrm{n}=106)$} \\
\hline Controlada & 50 & 8 & $16.0^{\mathrm{a}}$ \\
\hline No controlada & 56 & 11 & $19.6^{\mathrm{a}}$ \\
\hline \multicolumn{4}{|l|}{ Estrato etario $(n=104)$} \\
\hline$<1$ año & 18 & 2 & $11.1^{\mathrm{a}}$ \\
\hline 1 a más años & 86 & 17 & $19.8^{\mathrm{a}}$ \\
\hline \multicolumn{4}{|l|}{ Dieta $(n=106)$} \\
\hline Concentrado & 40 & 3 & $7.5^{\mathrm{a}}$ \\
\hline Incluye alimento casero & 66 & 16 & $24.2^{\mathrm{b}}$ \\
\hline \multicolumn{4}{|l|}{ Hábito (n=78) } \\
\hline Caza & 48 & 12 & $25.0^{\mathrm{a}}$ \\
\hline No caza & 30 & 7 & $23.3^{\mathrm{a}}$ \\
\hline \multicolumn{4}{|l|}{ Sexo $(n=106)$} \\
\hline Hembra & 55 & 14 & $25.5^{\mathrm{a}}$ \\
\hline Macho & 51 & 5 & $9.8^{\mathrm{b}}$ \\
\hline
\end{tabular}

a,b,c Letras diferentes dentro de cada variable indican asociación significativa $(p<0.05)$

\section{Análisis de Datos}

Se cuantificó el riesgo de infección (presencia de anticuerpos específicos anti-T. gondii) asociado al tipo de crianza mediante la prueba de Odds Ratio. La cuantificación de la asociación entre la presencia de serorreactores y las variables sexo, edad $(<1$, $>1$ año), dieta (concentrado y concentrado con alimento casero) y hábitos (caza, no caza) se evaluó mediante la prueba de Chicuadrado y, además, se obtuvo la razón de prevalencia para las variables contrastadas.

\section{Resultados}

La cuantificación de la variable en estudio (Crianza controlada vs. No controlada) como factor de riesgo para adquirir infeccio- nes por T. gondii mostró un Odds Ratio de 1.2 (0.47 - 3.50), no encontrándose asociación significativa (Cuadro 1).

Las variables sexo y dieta estuvieron significativamente asociadas con la presencia de serorreactores a T. gondii $(\mathrm{p}<0.05$, Cuadro 1). Las hembras tuvieron un 69\% mayor riesgo de infección por T. gondii que los machos, y aquellos gatos que consumen una dieta mixta tuvieron cuatro veces mayor riesgo que aquellos que consumían solo concentrado (Cuadro 2).

En términos generales, el 17.9\% (19/ 106) presentaron anticuerpos contra $T$. gondii; correspondiendo el 68.4\% (13/19) al estado agudo y el 31.6\% (6/19) al estado crónico de la enfermedad. 
Cuadro 2. Odds Ratio y razón de preval encia de infección por Toxoplasma gondii, según la variable de exposición. Lima, 2007

\begin{tabular}{|c|c|c|c|c|}
\hline & OR crudo & I.C. ${ }^{1}$ & $\begin{array}{l}\text { Razón de } \\
\text { prevalencia }\end{array}$ & I.C. \\
\hline \multicolumn{5}{|l|}{ Tipo de crianza } \\
\hline Controlada & 1 & & 1 & \\
\hline No controlada & 1.28 & $0.47-3.50$ & 1.23 & $0.54-2.81$ \\
\hline \multicolumn{5}{|l|}{ Estrato etario } \\
\hline$<1$ año & 1 & & & \\
\hline 1 a más años & 1.97 & $0.41-9.41$ & 1.78 & $0.450-7.03$ \\
\hline \multicolumn{5}{|l|}{ Dieta } \\
\hline Concentrado & 1 & & & \\
\hline Incluye alimento casero & 4.27 & $1.16-15.68$ & 3.48 & $1.08-11.22$ \\
\hline \multicolumn{5}{|l|}{ Hábito } \\
\hline No caza & 1 & & & \\
\hline Caza & 1.10 & $0.38-3.19$ & 1.07 & $0.48-2.42$ \\
\hline \multicolumn{5}{|l|}{ Sex 0} \\
\hline Macho & 1 & & & \\
\hline Hembra & 3.14 & $1.04-9.48$ & 2.60 & $1.01-6.70$ \\
\hline
\end{tabular}

${ }^{1}$ Intervalo de confianza al $95 \%$

\section{Discusión}

El riesgo de infección por T. gondii no reportó asociación significativa entre la crianza controlada y no controlada; sin embargo, se observó una ligera tendencia a la presencia de más casos de infección en gatos que tenían libre acceso a la calle. Araujo et al. $(1998,2003)$ mencionan que los gatos se infectan principalmente por la ingestión de quistes con bradizoítos presentes en los tejidos de hospederos intermediarios, como roedores y otros, como consecuencia del comportamiento innato de predadores. Asimismo, se dispone de estudios que indican que el 60\% de gatos serológicamente positivos al antígeno de $T$. gondii adquieren la infección por este medio (Cordero del Campillo et al., 1999); además, gatos que viven en las calles son más susceptibles a la exposición de ooquistes del medio ambiente y a la ingestión de pequeñas presas (Gauss et al., 2003).

En el presente estudio, no obstante, no se encontró que la actividad de caza se encuentre asociada a la presencia de infección. Ello puede deberse a que los propietarios ofrecieran respuestas negativas sobre los hábitos de caza de sus mascotas, probablemente porque no se hayan percatado o porque la infección se haya producido al estar en contacto con ambientes contaminados.

La variable dieta estuvo asociada significativamente con la frecuencia de infección con T. gondii, posiblemente debido a la costumbre de los propietarios y miembros de los hogares de proporcionar carne cruda o insuficientemente cocida que podrían con- 
tener quistes tisulares viables. Luzón et al. (1997) y Atias y Thiermann (1991) mencionan que la infección por bradizoítos contenidos en la carne y vísceras es una fuente importante de infección de toxoplasma, en especial para los felinos domésticos y silvestres.

La evaluación del sexo como variable de exposición presenta asociación significativa, donde las hembras presentan una mayor frecuencia de infección por T. gondii. Es posible que las hembras se dediquen a cazar pequeños mamíferos o pequeños vectores para dar de comer y enseñar a cazar a sus crías y esto podría representar un factor de riesgo para la infección; sin embargo, otros estudios demuestran una mayor tendencia en los machos sin llegar a encontrar una asociación significativa (Ovalle et al., 2000; Miro et al., 2004; Salant y Spira, 2004).

La edad es una variable asociada en diversos reportes a infección por T. gondii (Ovalle et al., 2000), especialmente en animales mayores de 2.5 años (Salant y Spira, 2004); aunque también se indica que no es necesariamente el factor edad sino el tiempo de exposición (Cordero del Campillo et al., 1999). Esto no se llega a corroborar en el presente estudio, donde la mayor frecuencia de serorreactores positivos en animales mayores de un año no llegó a ser estadísticamente significante.

Se utilizó la prueba de HAI como método diagnóstico, prueba rápida y sencilla para determinar si el gato estuvo en contacto con el $T$. gondii alguna vez en su vida. Por otro lado, la titulación con 2-mercaptoetanol ayuda a detectar el estado crónico y, de forma indirecta, el estado agudo. Un gato con serología positiva aguda indica que ha sufrido una infección y que ha estado eliminando ooquistes, los cuales esporulan de acuerdo a las condiciones favorables del ambiente, siendo fuente de infección para otras especies. La importancia médica en el estado crónico en el gato radica en que el gato puede volver a eliminar ooquistes si cursa con una enfermedad que deprima su sistema inmunológico.
Esta determinación es importante debido a que animales que eliminan ooquistes podrían representar una fuente de infección al hombre, aunque esta no es la ruta más importante. Acha y Szyfres (2003) señalan que el consumo de carne insuficientemente cocida es la principal fuente de infección para el humano.

\section{ConClusiones}

- No se encontró una asociación estadística entre el tipo de crianza del gato y la frecuencia de infección por Toxoplasma gondii.

- Se encontró una asociación significativa entre las variables sexo y dieta con la presencia de serorreactores a la infección por T. gondii.

\section{LiTERATURA CitADA}

1. Acha P, Szyfres B. 2003. Zoonosis y enfermedades transmisibles comunes al hombre y a los animales. $3^{\text {a }}$ ed. Publicación Científica № 580. Lima: Organización Panamericana de la Salud. 413 p.

2. Araujo W, Silva A, Langoni H. 1998. Toxoplasmose: uma zoonose - realidades e riscos. Rev Cães Gatos 13: 20-27.

3. Atias A, Thiermann E. 1991. Toxoplasmosis. En: Parasitología clínica. $3^{\text {a }}$ ed. Santiago de Chile: Ed Mediterráneo. p 269-281.

4. Araújo F, Silva N, Olicheski A, Beck C, Rodríguez R, Fialho C. 2003. Anticorpos para Toxoplasma gondii em soro de gatos internados no Hospital de Clínicas Veterinárias da UFRGS, Porto Alegre, RS, Brasil, detectados através da técnica de hemaglutinação indireta. Acta Sci Vet 31(2): 89-92.

5. Cordero del Campillo M, RojoVásquez F, Martínez-Fernández A, Sánchez M, Hernández S, Navarrete I, Díaz P, et al. 1999. Parasitología veterinaria. México DF: McGraw Hill Interamericana. 968 p. 
6. Dubey J, Lappin M. 2000. Toxoplasmosis y neosporosis. En: Enfermedades infecciosas en perros y gatos. $2^{\mathrm{a}}$ ed. México DF: McGraw Hill Interamericana. p 542-553.

7. Gauss C, Almeria S, Ortuño A, Garcia F, Dubey J. 2003. Seroprevalence of Toxoplasma gondii antibodies in domestic cats from Barcelona, Spain. J Parasitol 89: 1067-1068.

8. Lindsay D, Blagburn B, Dubey J. 1997. Feline toxoplasmosis and importance of the Toxoplasma gondii oocyst. Parasitol 19: 448-460.

9. Luzon M, Quintalla-Gonzalo A. 1997. Etiología y biología. En: Tratado de patología y producción ovina. Cap. 1. Ortego L (ed). Madrid: Ed Luzan. p 11-17.

10. Miró G, Montoya A, Jiménez S, Frizuelos $C$, Mateo M, Fuentes $I$. 2004. Prevalence of antibodies to Toxoplasma gondii and intestinal parasites in spray, faro and household cats in Spain. Vet Parasitol 126: 249-255.

11. Montoya J, Remington J. 2004. Toxoplasma gondii. En: Mandell,
Douglas, Bennett's. Enfermedades infecciosas, principios y práctica. $5^{\mathrm{a}} \mathrm{ed}$. Vol. 2. USA: Ed. Médica Panamericana. p 3452-3487.

12. Ovalle F, García A, Thibauth J, Lorca M. 2000. Frecuencia de anticuerpos anti-Toxoplasma gondii en gatos de la ciudad de Valdivia. Bol Chil Parasitol 55(3-4): 94-99.

13. Salant H, Spira D. 2004. Acrosssectional survey of anti-Toxoplasma gondii antibodies in Jerusalem cats. Vet Parasitol 124: 167-177.

14. Soulsby EJL. 1987. Parasitología y enfermedades parasitarias en los felinos domésticos. $7^{\text {a }}$ ed. México DF: Ed Interamericana. $823 \mathrm{p}$.

15. Tenter A, Heckeroth A, Weiss L. 2000. Toxoplasma gondii: from animals to humans. Int J Parasitol 12: 1217-1258.

16. Wiener Lab. 2000. Prueba de hemaglutinación indirecta (HAI) para la detección de anticuerpos contra Toxoplasma gondii. Manual de procedimiento e interpretación del Toxotest. Argentina: Wiener Lab. 6 p. 